\title{
The Pathogenesis of Xenobiotic-Induced Oxidative Stress in the Cardiac Microenvironment
}

Thomas SH ${ }^{1,4}$, Gipson N1, Vaughn J22, Omeler C ${ }^{3}$, Dawkins $\mathrm{NL}^{4}$ and

\section{Bradford $\mathrm{CN}^{1 *}$}

1Department of Biology, Tuskegee University, United States of America

2Department of Agricultural \& Environmental Sciences, Tuskegee University, United States of America

32Department of Biology, Oakwood University, United States of America

${ }^{4}$ Department of Food \& Nutritional Sciences, Tuskegee University, United States of America

*Corresponding author: Chastity Bradford, Associate Professor, Tuskegee University, 1200 West Montgomery Rd, Tuskegee Institute, AL, United States of America; Tel: 334-724-4797; E-mail: cbradford@tuskegee.edu

\section{Abstract}

The heart functions to ensure optimal perfusion of every organ while maintaining elasticity and compliance. Mainly composed of cardiac myocytes, fibroblasts, endothelial and vascular cells, the heart has the highest energy demand of all organs. Thus, it requires a high rate of adenosine triphosphate (ATP) production to maintain normal physiological function. Using oxidative phosphorylation, the heart produces ATP and concomitantly produces reactive oxidative species (ROS). Therefore, normal homeostasis and mitochondrial metabolism make the heart extremely susceptible to intrinsic and extrinsic oxidative stress. Exogenous foreign agents or aberrantly expressed endogenous molecules are characterized as cardiac xenobiotics (CX) which promote cardiac-specific toxicity. CX enter the body via nutritional and drug intake or environmental exposure and cause an imbalance in ROS production and antioxidant protection within the cardiac microenvironment. Chronic ROS exposure alters the cellular and molecular physiology of key detoxifying enzymes which modify cardiovascular structure and function. The heart has a tightly controlled antioxidant system that manages ROS and maintains homeostasis within the cardiac microenvironment. This strictly regulated system consists of endogenous enzymes such as superoxide dismutase (SOD), catalase (CAT) and glutathione peroxidase (GPx) and exogenous antioxidants such as vitamins, minerals, polyphenols and carotenoids derived from nutritional sources. Sustained levels of cardiac xenobiotics can result in chronically imbalanced ROS production that outpaces the antioxidant system. Over time this imbalanced system results in irreversible cellular and subcellular damage, altering cardiac 
structure and function and increasing the risk of cardiac dysfunctions such as maladaptive left ventricular hypertrophy, cardiac fibrosis and heart failure.

Keywords: Xenobiotics; Cardiac; Reactive Oxidative Species (ROS); Detoxifying Enzymes; Antioxidants

Abbreviations: ATP: Adenosine Triphosphate; ROS: Reactive Oxidative Species; SOD: Superoxide Dismutase; GPx: Glutathione Peroxidase; CX: Cardiac Xenobiotics

\section{Introduction}

Maintenance of cardiac output to ensure adequate perfusion of the heart itself and every organ in the body is the ultimate function of the heart. The heart is a compliant and elastic organ with both mechanical and electrophysiological functions. It is mostly composed of cardiac myocytes (highest volume of mitochondria), cardiac fibroblasts, mast cells, endothelial cells, and vascular cells. In addition to its cellular composition, the cardiac microenvironment is composed of subcellular organelles and macromolecules, which play a vital role in adenosine triphosphate (ATP) production. ATP production and enzymatic synthesis are critical for proper signal transduction, cell to cell communication, and maintenance of cellular membrane integrity. The heart has a high metabolic demand; thus, it continuously produces ATP via oxidative phosphorylation, which creates the highest rate of production of reactive oxidative species (ROS) [1]. This demand, coupled with low levels of cardiac detoxifying enzymes, makes the heart extremely susceptible to oxidative stress. ROS and key detoxifying enzymes are primarily produced and located within the mitochondria, where cardiac cellular metabolism occurs. Mitochondrial ATP production produces hydroperoxyl, hydroxyl, peroxynitrite radicals, superoxide anion, hydrogen peroxide and carbon dioxide [2]. In addition to this intrinsic oxidative stress, the heart is susceptible to high levels of external oxidative stress from hypertension-induced pressure overload, cardiac toxicants, and xenobiotics. Therefore, homeostatic regulation of cardiac metabolism requires a tightly regulated microenvironment with a cardiac-specific antioxidant system. Detoxifying enzymes such as superoxide dismutase (SOD), glutathione peroxidase (GPx), and catalase (CAT), are part of the first line defense against ROS-mediated cardiac injury [3]. Endogenous mechanisms within the cardiac microenvironment and exogenous antioxidant mechanisms from natural sources can modulate ROS production and oxidative stress derived from metabolic and detoxifying activity. High metabolic demand increases cardiac susceptibility to oxidative stress of cardiac toxicants and xenobiotics.

\section{Cardiac Xenobiotics and Oxidative Stress}

Cardiac xenobiotics (CX) are exogenous foreign agents or aberrantly expressed endogenous compounds which promote cardiac-specific toxicity [4]. The primary modes of cardiac xenobiotic entry are achieved via the environment, nutrition, and/or drug exposure. CX drugexposure's wide range of classes include anticancer drugs (i.e. doxorubicin), ethanol, psychoactive drugs (i.e. methylenedioxymethamphetamine a.k.a. "ecstasy"), and catecholamines [5]. Both pulmonary and extrapulmonary CX environmental exposure have demonstrated decreases in cardiac heart rate variability. The inhalation of toxic particles extends its damage to cardiovascular tissue promoting cardiac arrythmias, altering cardiac repolarization, and increasing blood pressure [6]. Nutrition also exposes the heart to oxidants, and it has been well established that diets high in trans and saturated fats promote cardiovascular disease (CVD). Research shows that different types of athletes exhibit different xenobiotic profiles based upon diet, designed to enhance performance and recovery [7]. Based upon the amount consumed and physiological and/or pathological state of the consumer, nutrition can be beneficial and/or deleterious. Thus, there is an association between CX exposure, normal cardiovascular physiology and increased prevalence of CVD [8].

Reactive oxidative species are free radicals and nonradical derivatives produced as a by-product of cellular metabolism; and at low levels promote cell growth, but at high levels can promote tissue injury and reduce cell function. Under physiological conditions, ROS production, detoxifying enzymes (i.e. SOD), and antioxidants (i.e. carotenoids) carefully balance intracellular ROS to maintain cellular homeostasis [9]. Under pathological conditions, there is an increase in pro-oxidant to antioxidant ratio; mitochondrial energy metabolism is altered; and the heart reverts to fetal metabolism switching from beta-oxidation to glucose oxidation. Various biomarkers of oxidative stress provide indirect evidence for the effect of ROS in CVD. For example, the 
overexpression of GPx indicates the capacity to detoxify ROS prevents myocardial infarction, myocyte hypertrophy, and fibrosis [3]. Increased levels of 8hydroxy-2'-deoxyguanosine, a marker of ROS-induced oxidative damage, are also present in CVD [10]. Increased ROS production that is not counterbalanced by antioxidants can alter cellular homeostasis and cause deleterious genetic and epigenetic mutations that are responsible for cell cycle regulation and antioxidant enzyme protein synthesis [11]. Oxidative stress can alter the backbone and side chains of proteins, and these structural alterations promote functional changes that alter the integrity of macromolecules, affect cell membrane permeability, and exacerbate oxidative damage [12,13]. Chronic oxidative stress results in irreversible and fatal damage of macromolecules which promotes maladaptive cardiac hypertrophy, extensive cardiac fibrosis and heart failure [14].

\section{Conclusion}

In a normal physiological state, cardiac mitochondrial metabolism is accompanied by concomitant ROS production, which requires a strictly regulated antioxidant system to maintain homeostasis and prevent irreversible damage of macromolecules within the cardiac microenvironment. CX-induced toxicity generates excessive ROS production and creates a harmful imbalance of the regulatory system, causing cardiacspecific oxidative stress. The primary endogenous systems use key detoxifying enzymes such as SOD, GPx and CAT to regulate ROS production and exogenous antioxidants consisting of vitamins, minerals, polyphenols and/or carotenoids derived from nutritional sources to maintain homeostasis.

Unregulated CX exposure can invoke irreversible damage of macromolecules in the cardiac microenvironment promoting a sequence of events leading to maladaptive cardiac remodeling, which negatively impairs cardiac contractility and compliance. Thus, it remains essential to clearly characterize the endogenous and exogenous antioxidants responsible for managing cardiac oxidative stress; determine the role of dietary intervention on ROS production; and to provide alternative strategies to maintaining optimal protection against CX-induced oxidative stress.

\section{References}

1. Shah AM, Channon KM (2004) Free Radicals and Redox Signaling in Cardiovascular Disease. Heart 90(5): 486-487.
2. Meij JT, Haselton CL, Hillman KL, Muralikrishnan D, Ebadi M, et al. (2004) Differential Mechanisms of Nitric Oxide- and Peroxynitrite-Induced Cell Death. Mol Pharmacol 66(4): 1043-1053.

3. Shiomi T, Tsutsui H, Matsusaka H, Murakami K, Hayashidani S, et al. (2004) Overexpression of Glutathione Peroxidase Prevents Left Ventricular Remodeling and Failure after Myocardial Infarction in Mice. Circulation 109(4): 544-549.

4. Costa VM, Carvalho F, Duarte JA, Bastos M de L, Remião F (2013) The Heart as a Target for Xenobiotic Toxicity: The Cardiac Susceptibility to Oxidative Stress. Chem Res Toxicol 26(9): 1285-1311.

5. Croom E (2012) Metabolism of Xenobiotics of Human Environments. Prog Mol Biol Transl Sci 112: 31-88.

6. Liao D, Shaffer ML, Rodriguez-Colon S, He F, Li X, et al. (2010) Acute Adverse Effects of Fine Particulate Air Pollution on Ventricular Repolarization. Environ Health Perspect 118(7): 1010-1015.

7. Al-Khelaifi F, Diboun I, Donati F, Botrè F, Alsayrafi M, et al. (2018) Metabolomics Profiling of Xenobiotics in Elite Athletes: Relevance to Supplement Consumption. J Int Soc Sport Nutr 15(1): 48.

8. Pai VB, Nahata MC (2000) Cardiotoxicity of Chemotherapeutic Agents: incidence, treatment and prevention. Drug Saf 22(4): 263-302.

9. Taverne YJHJ, Bogers AJJC, Duncker DJ, Merkus D (2013) Reactive Oxygen Species and the Cardiovascular System. Oxid Med Cell Longev 2013: 1-15.

10. Kroese LJ, Scheffer PG (2014) 8-Hydroxy-2'deoxyguanosine and Cardiovascular Disease: A Systematic Review. Curr Atheroscler Rep 16(11): 452.

11. Jiang D, Rusling JF (2019) Oxidation Chemistry of DNA and p53 Tumor Suppressor Gene. ChemistryOpen 8(3): 252-265.

12. Davies MJ, Fu S, Wang H, Dean RT (1999) Stable Markers of Oxidant Damage to Proteins and their Application in the Study of Human Disease. Free Radic Biol Med 27(11-12): 1151-1163.

13. Ramana KV, Srivastava S, Singhal SS (2017) Lipid Peroxidation Products in Human Health and Disease 2016. Oxid Med Cell Longev 2017: 1-2. 


\section{Advances in Clinical Toxicology}

14. Basu U, Case AJ, Liu J, Tian J, Li YL, et al. (2019) Redox-Sensitive Calcium/Calmodulin-Dependent

Signaling and Hypertension. Redox Biology 2019: Protein Kinase II $\alpha$ in Angiotensin II Intra-Neuronal 101230. 